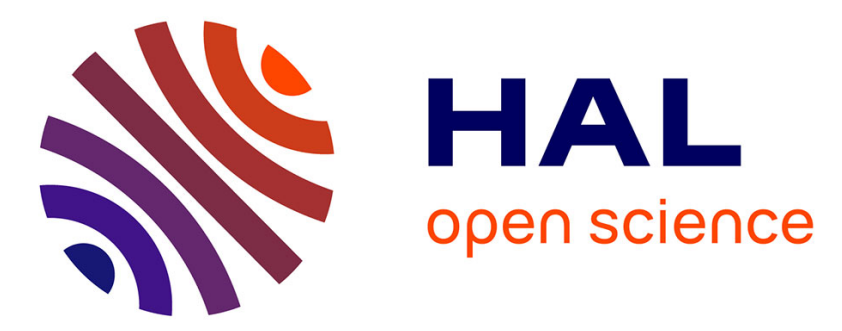

\title{
Stokes flow through a Boolean model of spheres: Representative volume element
}

Bassam Abdallah, François Willot, Dominique Jeulin

\section{To cite this version:}

Bassam Abdallah, François Willot, Dominique Jeulin. Stokes flow through a Boolean model of spheres: Representative volume element. Transport in Porous Media, 2015, 109 (3), pp.711-726. $10.1007 / \mathrm{s} 11242-015-0545-2$. hal-01232510

HAL Id: hal-01232510

https://hal-mines-paristech.archives-ouvertes.fr/hal-01232510

Submitted on 23 Nov 2015

HAL is a multi-disciplinary open access archive for the deposit and dissemination of scientific research documents, whether they are published or not. The documents may come from teaching and research institutions in France or abroad, or from public or private research centers.
L'archive ouverte pluridisciplinaire HAL, est destinée au dépôt et à la diffusion de documents scientifiques de niveau recherche, publiés ou non, émanant des établissements d'enseignement et de recherche français ou étrangers, des laboratoires publics ou privés. 


\title{
Stokes flow through a Boolean model of spheres: Representative Volume Element
}

\author{
B. Abdallah ${ }^{1}$. F. Willot ${ }^{1}$. D. Jeulin ${ }^{1}$
}

"Postprint" document (three remaining typos in the published version herein corrected in red, pp. 6, 11 and 14; results unchanged).

\begin{abstract}
The Stokes flow is numerically computed in porous media based on 3D Boolean random sets of spheres. Two configurations are investigated in which the fluid flows inside the spheres or in the complementary set of the spheres. Full-field computations are carried out using the Fourier method of Wiegmann (2007). The latter is applied to large system sizes representative of the microstructure. The overall permeability of the two models as well as the Representative Volume Element (RVE) are estimated as a function of the pore volume fraction. We give numerical estimates for the asymptotic behavior of the permeability in the dilute limit for the solid phase, and close to the percolation threshold of the pores. FFT maps of the velocity field are presented, for increasing values of the pore volume fraction. The patterns of the local velocity field is analysed using various morphological criteria. The tortuosity of the streamlines is found to be much higher than the geometrical tortuosity, for both models. The histograms of the velocity field are computed at increasing pore volume fraction. The covariance of orientation is used to characterize the spatial correlation of the velocity field.
\end{abstract}

Keywords Porous media - Stokes flow · FFT methods · Representative Volume Element · Tortuosity · Streamlines

\section{Introduction}

Since the pioneering works of Carman (1937), the permeability of random media has been a long-standing problem. In some early works, estimates for

\footnotetext{
1 Mines ParisTech, PSL Research University, Center for Mathematical Morphology, 35 rue $\mathrm{S}^{\mathrm{t}}$-Honoré, 77300 Fontainebleau, France.

Corresponding author: F. Willot, E-mail: francois.willot@mines-paristech.fr, Tel.: +33 164 6947 06, Fax.: +33164694707
} 
the permeability of flow around spheres have been derived making use of periodic structures (Richardson and Zaki, 1954; Martin et al, 1951) or of concentric sphere models (Happel, 1958; Kuwabara, 1959). Other studies have focused on dilute, random suspensions of spheres (Childress, 1972; Howells, 1974; Hinch, 1977) or on periodic arrays (Zick and Homsy, 2006; Sangani and Acrivos, 1982; Cheng and Papanicolaou, 1997). Another approach, based on variational formulations, consists in deriving rigorous bounds for permeability (Doi, 1976; Rubinstein and Torquato, 1989; Torquato and Lu, 1990). These bounds take into account two-point correlations functions as well as the so-called "surfacesurface" and "surface-void" correlation functions. Interestingly, earlier bounds made use of three-point correlation functions (Prager, 1961; Weissberg and Prager, 1970). More recently, self-consistent schemes for the effective permeability have also been proposed (Boutin, 2000; Koo and Sangani, 2002). We refer to (Torquato, 2002; Dormieux et al, 2006) for an overview on the subject.

Among numerical methods for the computation of the effective permeability, finite-difference schemes (Martys et al, 1994) and, more recently, fast multipole expansions (Koo and Sangani, 2002) as well as Boltzmann Lattice Methods (Belov et al, 2004; Lee and Lee, 2013) have been used. Recently, largescale computations have been carried out using a finite element method (Karim et al, 2014). Interestingly, several Fourier-based algorithms have been devised to treat Stokes flow (Wiegmann, 2007; Monchiet et al, 2009; N'guyen et al, 2013; Bignonnet and Dormieux, 2014). Such Fourier-based numerical methods solve periodic Stokes flow problems, which are based on a rigorous homogenization theory (Ene and Sanchez-Palencia, 1975; Matheron, 1965, 1966). The FFT method of (Wiegmann, 2007) has been used to predict the effective permeability of open foam structures, using 3D images (Redenbach et al, 2011). However, the local velocity and pressure fields occuring in porous random microstructures has not been extensively studied in 3D. The "Representative Volume Element" (RVE) has been tackled elasticity and conductivity for polycrystals (Kanit et al, 2003), Boolean models (Willot and Jeulin, 2009) and multiscale materials (Willot and Jeulin, 2010), making use of Matheron's theory of regionalized variables (Matheron, 1971). Other recent works include optics (Azzimonti et al, 2013) and acoustic (Peyrega and Jeulin, 2013). Results are also provided in (Du and Ostoja-Starzewski, 2006) for Stokes flow in a 2D hard-core models of discs, however the subject has not been studied to the same extent for permeability in random media.

This work focuses on the slow motion of a fluid past a random set of obstacles. It is organized as follows. The Stokes equation and microstructure of interest are given in Sec. (2), where we also present the numerical Fourier-based method. The Representative Volume Element (RVE) for the permeability is studied in Sec. (4). The microstructure permeability is computed in the entire range of pore volume fraction in Sec. (5). The asymptotic behaviour in the dilute and percolation limit is studied in Sec. (6). The streamlines, their tortuosity, the velocity field's histograms and covariances are computed in Sec. (7). 


\section{Stokes Flow in porous media}

We consider a domain $V$ made of a porous $(P)$ and solid $(S)$ phase. We note $f=V_{V}=|P| /|V|$ the pore volume fraction and $1-f=1-V_{V}=|S| /|V|$ the volume fraction of the solid phase. For simplicity, we take $V=[-L / 2 ; L / 2]^{3}$, a cubic domain of side $L$ aligned with the axis $\mathbf{e}_{k}(k=1,2,3)$ of a Cartesian coordinates system. We are interested in the steady-state flow of an incompressible Newtonian fluid in the pores. The fluid velocity vector $\mathbf{u}(\mathbf{x})$ is defined in the pores where it follows the Stokes equation:

$$
\mu \Delta u_{i}(\mathbf{x})=\partial_{i} p(\mathbf{x}), \quad \partial_{i} u_{i}(\mathbf{x})=0 \quad(\mathbf{x} \in P),
$$

where $\mu$ is the fluid viscosity, measured in $\mathrm{Pa} \cdot \mathrm{s}, p(\mathbf{x})$ is the pressure field at point $\mathbf{x}$, measured in $\mathrm{Pa}$ and $\Delta$ is the Laplacian operator. At the pore-solid interface $\partial S$, the fluid satisfies the no-slip boundary condition:

$$
\mathbf{u}(\mathbf{x})=0 \quad(\mathbf{x} \in \partial S)
$$

By convention, the domain of definition of the fluid is extended to the solid phase as:

$$
\mathbf{u}=0 \quad(\mathbf{x} \in S) .
$$

Along the boundary of the domain, periodic boundary conditions for the fluid velocity are applied:

$$
\mathbf{u}\left(\mathbf{x} \pm \mathbf{e}_{k} L\right) \equiv \mathbf{u}(\mathbf{x}) .
$$

A macroscopic pressure gradient of intensity $\delta P$ along the direction $\mathbf{E}$ is applied:

$$
\left\langle\partial_{i} p(\mathbf{x})\right\rangle=\delta P E_{i}, \quad|\mathbf{E}|=1,
$$

where $\langle\cdot\rangle$ stands for the average over the domain $V$. The boundary conditions for the pressure field specifies that its fluctuations $p^{*}$ are periodic (Ene and Sanchez-Palencia, 1975):

$$
p^{*}(\mathbf{x})=p(\mathbf{x})-\delta P(\mathbf{x} \cdot \mathbf{E})=p^{*}\left(\mathbf{x} \pm L \mathbf{e}_{k}\right) .
$$

The permeability tensor $\kappa_{i j}$, measured in $\mathrm{m}^{2}$, is defined at the macroscopic scale by Darcy's law:

$$
\left\langle u_{i}(\mathbf{x})\right\rangle=-\frac{\kappa_{i j}}{\mu}\left\langle\partial_{j} p(\mathbf{x})\right\rangle=-\frac{\kappa_{i j} E_{j}}{\mu} \delta P .
$$

In the remaining part of this work, we assume that the porous phase is macroscopically isotropic. By convention, we choose $\mathbf{E}=\mathbf{e}_{1}$ so that the permeability tensor $\kappa$ is identified with its component $\kappa=\kappa_{11}$ and:

$$
\kappa=-\frac{\mu\left\langle u_{1}(\mathbf{x})\right\rangle}{\delta P} .
$$

The permeability is independent of the fluid viscosity. 
Equations. (1)-(6) are solved numerically using the iterative Fourier-based scheme of Wiegmann (2007). The velocity and pressure field are discretized on a $3 \mathrm{D}$ grid of voxels. The method consists in rewriting the Stokes flow problem as a set of four Poisson equations which are discretized by centered differences. Artificial forces are added along solid surfaces to enforce the no-slip condition (2). Each component of the local velocity vector is interpreted as the flow of the fluid across voxel faces. The gradient and Laplacian operators are discretized by finite differences between adjacent voxels. The system of equations is solved iteratively using the generalized minimal residual method for linear systems (Saad and Schultz, 1986). Finite differences are computed in the Fourier domains using the shift operator. The method uses 3D microstructure images, and no meshing is necessary, which allows one to treat arbitrarily complex geometries.

The permeability of the medium is computed numerically by setting $\delta P=1$ [Pa], averaging on the velocity field $u_{1}(\mathbf{x})$ and using Eq. (8). By convention, we also fix $\mu=1[\mathrm{~Pa} \cdot \mathrm{s}]$. In (Wiegmann, 2007), convergence is achieved using minimal residual method (MINRES). At each iterations, Fourier transforms are used to invert the Laplacian operator. In the limit of a very high number of iterations, the fluid velocity is zero inside the obstacles. In practice, iterations are stopped when the following two conditions are met:

$$
\begin{gathered}
\max _{\mathbf{x} \in S}\|\mathbf{u}(\mathbf{x})\| \leq \eta_{1} \max _{\mathbf{x} \in P}\|\mathbf{u}(\mathbf{x})\| \\
\langle\|\mathbf{u}(\mathbf{x})\|\rangle_{S} \leq \eta_{2}\langle\|\mathbf{u}(\mathbf{x})\|\rangle_{P}
\end{gathered}
$$

where $\langle\cdot\rangle_{S, P}$ are averages over the solid and porous phases and $\eta_{1}=10^{-3}$, $\eta_{2}=10^{-4}$.

In the following, we also compare our numerical results with analytical bounds and estimates. We consider the Carman-Kozeny estimates (Carman, 1937; Kozeny, 1927):

$$
\kappa \approx \kappa^{\mathrm{CK}}=\frac{f^{3}}{c \gamma^{2}},
$$

where $\gamma=|\partial S| /|V|$ is the specific surface area, or surface area density, measured in $\mathrm{m}^{-1}$, and $c$ is an empirical constant. This adjustable parameter takes into account the shape of particles in random media. For the Boolean model, we fix it to $c=5$ (Happel, 1958). For comparison purpose, the upper-bound $\kappa^{\mathrm{UB}}$ proposed in (Doi, 1976) is considered:

$$
\kappa^{\mathrm{UB}}=\frac{2}{3} \int_{0}^{\infty} \mathrm{d} h\left[\frac{f^{2}}{\gamma^{2}} F_{S S}(h)-\frac{2 f}{\gamma} F_{S P}(h)+F_{P P}(h)\right],
$$

where $F_{S S}, F_{S P}$ and $F_{P P}$ are the solid-solid, fluid-solid and solid-solid surface correlation functions, respectively. They are readily computed using the method given in (Doi, 1976). The histograms $P_{u_{i}}$ of the components of the velocity field read:

$$
P_{u_{i}}(t)=\frac{1}{P} \int_{P} \mathrm{~d}^{3} \mathbf{x} \delta\left(t-u_{i}(\mathbf{x})\right)
$$


They are numerically computed from full-fields FFT maps. The latter also provide the tortuosity $\tau$ of the fluid streamlines which is defined by:

$$
\tau=\frac{L_{f}}{L}
$$

In the above, $L$ is the distance between two opposite faces in the domain, and $L_{f}$ is the mean length of the fluid paths which connect two points located at opposite faces. For comparison purposes, we also estimate the geometrical tortuosity:

$$
\tau_{G e o}=\frac{L_{f}^{G e o}}{L},
$$

defined from the length $L_{f}^{G e o}$ of geodesics in the porous phase. The latter are the shortest paths in the pores that connect two opposite sides of the domain. Another quantity of interest is the static viscous tortuosity $\tau_{0}$ (Johnson et al, 1982, 1987; Perrot et al, 2008) related to the fluctuations of the fluid local velocity by:

$$
\tau_{0}=\frac{\left\langle\mathbf{u}^{2}\right\rangle}{\langle\mathbf{u}\rangle^{2}},
$$

with $\left\langle\mathbf{u}^{2}\right\rangle=\left\langle u_{i} u_{i}\right\rangle$ and $\langle\mathbf{u}\rangle^{2}=\left\langle u_{i}\right\rangle\left\langle u_{i}\right\rangle$. The tortuosity factor given in (Matyka and Koza, 2012) is similarly defined as:

$$
\tau_{\text {Matyka }}^{\mathrm{A}, \mathrm{B}}=\frac{\langle\|\mathbf{u}\|\rangle}{\left\langle u_{1}\right\rangle} .
$$

Finally, we estimate the correlation of orientations of the local velocity field from Altendorf and Jeulin (2011) using the following morphological criterion, based on scalar products:

$$
C_{\alpha}(h)=\int_{\mathbf{x} \in V} \mathrm{~d}^{3} \mathbf{h} \frac{(\mathbf{u}(\mathbf{x}) \cdot \mathbf{u}(\mathbf{x}+\mathbf{h}))^{2}}{\|\mathbf{u}(\mathbf{x})\|^{2}\|\mathbf{u}(\mathbf{x}+\mathbf{h})\|^{2}} .
$$

\section{Boolean model of spheres}

In the remaining part of this work, is considered a Boolean model of spheres (Matheron, 1967) of constant diameter $D>0$. In this model, the sphere centers are located according to a Poisson point process in $V$, so that spheres are allowed to overlap. The model is parametrized by the density of spheres centers or equivalently by the spheres volume fraction. We denote $1-q$ the spheres volume fraction and $q$ the volume fraction of the embedding medium. Two types of models are considered where spheres are either "solid" or "porous". In model (A) flow occurs solely outside the spheres whereas in model (B) flow occurs inside spheres only. Therefore, we have $f=q$ for model (A) and $f=1-q$ for model (B). We denote the permeability of these models by $\kappa_{A}$ and $\kappa_{B}$ respectively. 
The specific surface area $\gamma$ is obtained from:

$$
\gamma=-\left.4 \frac{\partial C(h)}{\partial h}\right|_{h=0}
$$

where $C(h)$ is the covariance function (Matheron, 1967). In a Boolean model of spheres (Matheron, 1967) we have for models (A) and (B), respectively:

$$
C_{A}(h)=q^{1+\frac{3 h}{2 D}-\frac{h^{3}}{2 D^{3}}}, \quad C_{B}(h)=1-2 q+q^{1+\frac{3 h}{2 D}-\frac{h^{3}}{2 D^{3}}} .
$$

For both models the specific surface area is:

$$
\gamma=-\frac{6 q}{D} \log q
$$

Therefore the Carman-Kozeny estimates (Eqs. 11) for models (A) and (B) read, respectively:

$$
\kappa^{\mathrm{CK}-\mathrm{A}}=\frac{f D^{2}}{180 \log ^{2}(f)}, \quad \kappa^{\mathrm{CK}-\mathrm{B}}=\frac{f^{3} D^{2}}{180(1-f)^{2} \log ^{2}(1-f)} .
$$

In the following, the Boolean model is discretized over a 3D grid of $L \times \mathrm{L} \times L$ voxels, with increasing size $L=100,256,512$ (Fig. 1). The spheres diameter $D$ is equal to $D=15$ voxels. This makes sure the particles ressemble spheres, as shown in the enlarged 3D view in Fig. (1). Hereafter, by convention, all lengths are rescaled by the spheres radius $R$, or equivalently, we take $D=2 R=2[\mathrm{~m}]$. Accordingly, microstructures contain an increasing number of spheres when $L$ increases. The entire range of pore volume fraction $f_{c}^{A} \leq f \leq 1$ for model (A) and $f_{c}^{B} \leq f \leq 1$ for model (B) is considered, where $f_{c}^{B}$ and $f_{c}^{A}$ are the percolation threshold of the spheres and of its complementary, respectively. The following approximate values are available: $f_{c}^{B} \approx 0.2895$ (Rintoul and Torquato, 1997) whereas $f_{c}^{A}$ ranges from 0.0317 (van der Marck, 1996; Priour Jr, 2014) to 0.0540 (Jeulin and Moreaud, 2006) according to different authors.

Note that the spheres are reproduced periodically in all directions without further modification. In particular, some spheres are completely surrounded with the fluid in model $A$.

\section{Representative Volume Element}

The effective permeability is estimated by the apparent permeability $\kappa$, computed by averaging on the velocity field (7). This estimation gives the exact permeability only in the limit of very large system sizes $L \rightarrow \infty$. In a volume $V$ of finite size, the relative error $\epsilon$ of the estimate for the permeability is given by:

$$
\epsilon=\frac{2 D_{\kappa}(V)}{\sqrt{n} \kappa}
$$

where $D_{\kappa}^{2}(V)$ is the variance of the apparent permeability over $n$ random realizations of volume $V$. Alternatively, using a single large realization of size 


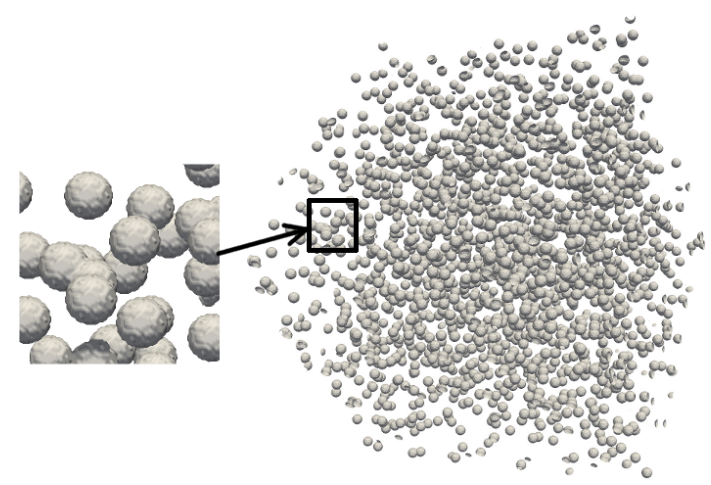

Fig. 1 Boolean model of spheres discretized on a grid of $512^{3}$ voxels. Spheres diameter: 15 voxels; Spheres volume fraction: $10 \%$.

$V$, we compute an estimate of $D_{\kappa}^{2}\left(V^{\prime}\right)$ for all volumes $V^{\prime}<V$ by considering disjoint subdomains $V^{\prime} \subseteq V$. The asymptotic expansion of $D_{\kappa}^{2}\left(V^{\prime}\right)$ when $V \gg$ $V^{\prime}$ and $V^{\prime} \rightarrow \infty$ reads (Matheron, 1971):

$$
\frac{D_{\kappa}^{2}\left(V^{\prime}\right)}{D_{\kappa}^{2}} \sim A_{3}^{\kappa}\left(\frac{1}{V^{\prime}}-\frac{1}{V}\right)
$$

where $A_{3}^{\kappa}$ is the integral range for the permeability (Kanit et al, 2003). In the above, the term $1 / V$ represents a lower-order correction compared to $1 / V^{\prime}$. The point variance $D_{\kappa}^{2}=\left\langle u_{1}^{2}\right\rangle-\left\langle u_{1}\right\rangle^{2}$ is numerically estimated using the velocity fields $u_{1}(\mathbf{x})$. In practice, the term $1 / V$ can be neglected and we have:

$$
\frac{D_{\kappa}^{2}\left(V^{\prime}\right)}{D_{\kappa}^{2}} \sim \frac{A_{3}^{\kappa}}{V^{\prime}}
$$

Likewise, fluctuations are encountered when estimating the pore volume fraction in a volume $V$. The latter scales as:

$$
\frac{D_{f}^{2}(V)}{f(1-f)} \sim \frac{A_{3}^{f}}{V}, \quad V \gg A_{3}^{f},
$$

where we refer to $A_{3}^{f}$ as the integral range for the microstructure. For model (B) (Matheron, 1967):

$$
A_{3}^{f}(f)=\int_{|\mathbf{h}| \leq 2 R} \frac{f^{1+\frac{3 h}{2 D}-\frac{h^{3}}{2 D^{3}}}-f^{2}}{f(1-f)} d^{3} \mathbf{h},
$$

for model (A) $f$ must be replaced by $1-f$ in relation (27).

The point-variance $D_{\kappa}^{2}$ is numericaly obtained by computing second moments of the velocity field over the voxels grid. The variances $D_{\kappa}^{2}\left(V^{\prime}\right)$ are computed by first subdividing a large volume $V$ into a set of non-overlapping subdomains, each of size $V^{\prime}$. In practice, cuboidal domains are considered and 


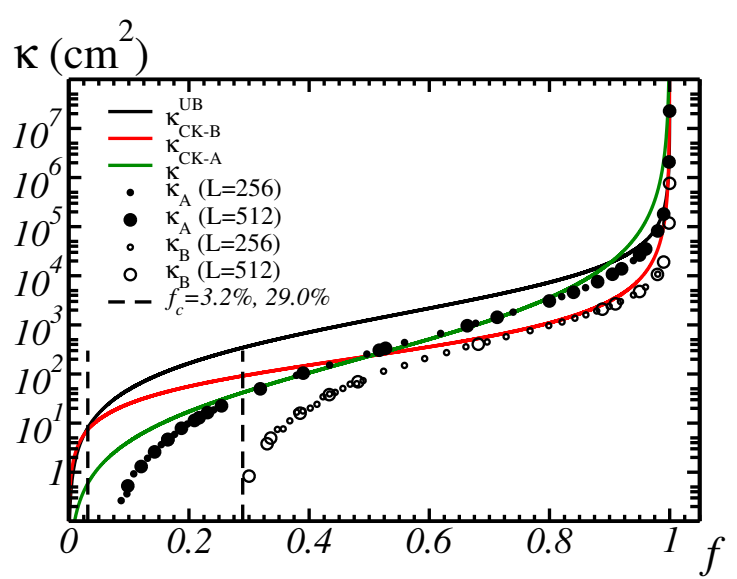

Fig. 2 Effective permeability $\kappa^{A, B}$ of the Boolean models (A) and (B) (disks and circles) with respect to the pore volume fraction $f$, at two resolutions. Solid lines: Carman-Kozeny estimate $\kappa^{C K}$ and upper-bound $\kappa^{U B}$.

the apparent permeability in each subdomain is given by the mean of the velocity field $u_{1}$ over each region. Accordingly, when the volume $V^{\prime}$ is small, the variance is computed over a large data set. Conversely, the variance $D_{\kappa}^{2}\left(V^{\prime}\right)$ corresponding to the maximum volume size $V^{\prime}=V / 8$ is computed over a set of 8 values.

\section{Permeability of the Boolean models}

The effective permeability is estimated for increasing pore volume fraction $f$ in the range $\left[f_{c}^{A} ; 1\right]$ for model $(\mathrm{A})$ and $\left[f_{c}^{B} ; 1\right]$ for model $(\mathrm{B})$. Results are plotted in Fig. 2 in lin-log scale. As expected, the permeability is largest for the complementary model of spheres (B), with lower percolation threshold than in model (A). Both models show a convexity change in lin-log scale at about $65 \%$ for model (B) and $50 \%$ of pore volume fraction for model (A).

Furthermore, the estimate $\kappa^{\mathrm{CK}-\mathrm{A}}$ (shown in green in Fig. 2) is close to FFT predictions in the range $0.3<f<0.8$. Outside of this region, the estimate differs noticeably from the FFT data. As reported in (Happel, 1958), the Carman-Kozeny estimate (11) with $c=5$ agrees well with experimental data in the range $0.26<f<0.8$ for particles of various shapes maintained in a fixed position. This indirectly confirms the validity of the FFT computations for Stokes flow. Interestingly, the Carman-Kozeny estimate $\kappa^{\mathrm{CK}-\mathrm{B}}$ for model B (red curve) is close to our FFT predictions in a different range of porosity, namely $0.7<f<0.9$. In this domain, the regions surrounding the spheres are mostly disconnected, and the solid phase is akin to a set of random fixed particules with non-spherical shape. In the dilute regime $f \rightarrow 1$, as expected, the Carman-Kozeny estimate fails for all models. 
FFT fields are also used to study the representative volume element for the permeability as a function of the pore volume fraction $f$. The integral range $A_{3}^{\kappa}$ is estimated numerically by fitting the left-hand side of (25) with a scaling law $D_{\kappa}^{2}\left(V^{\prime}\right) \sim 1 / V^{\prime}$. As expected, the latter is recovered when $V$ is large enough. This is illustrated in Fig. 3 where the variance of the apparent permeability $D_{\kappa}^{2}(V)$ of model B with $f=0.68$ is represented as a function of $V$. For a requested precision $\epsilon$, the corresponding RVE size is obtained using (23) and the previously determined integral range.

In Fig. 4 the relative precision is fixed to $\epsilon=5 \%$. We set $V=\ell^{3}$ and plot the RVE lengths $\ell_{\kappa}^{A}$ and $\ell_{\kappa}^{B}$ for the permeability in models (A) and (B) respectively, as a function of $f$. The microstructure RVE lengths $\ell_{f}^{A}$ and $\ell_{f}^{B}$ at the same relative precision are plotted for comparison. For both models, the RVE size for the permeability strongly increases when approaching the percolation threshold. Strong microstructural effects are especially important for model B. Using the discretization $D=15$ voxels, a system with $1350^{3}$ voxels is required to estimate the permeability $\kappa^{B}$ at $5 \%$ relative error when $f \approx 0.32$.

The RVEs for permeability appear to be significantly higher than in elasticity or thermal conductivity (Kanit et al, 2003; Willot and Jeulin, 2009). It is also useful to compare these results with numerical data obtained for a Stokes flow around randomly-distributed, non-overlapping discs (Du and Ostoja-Starzewski, 2006). The authors studied the convergence of the apparent permeability with respect to the size of the computational domain. Highest convergence rates were obtained at lowest porosity (50\%). Conversely, the worst convergence rates were observed at highest porosity $(80 \%)$. In $3 \mathrm{D}$, the RVE for model $A$ is quite insensitive to the porosity in the range $0.5<f<0.8$, but increases with $f$ in the dilute regime $f>0.8$ (Fig. 4). The behavior observed in $2 \mathrm{D}$ for non-overlapping discs is accordingly qualitatively recovered in 3D for the Boolean model, but for a different range of porosity. Interestingly, (Du and Ostoja-Starzewski, 2006) link the increase of the RVE with that of the size of the channels located in-between obstacles.

\section{Dilute limit and behaviour near the percolation thresholds}

We first consider the behavior of the permeability in models (A) and (B) in the dilute limit $f \rightarrow 1$, i.e. for a small volume fraction of obstacles. Numerical FFT data are represented in log-log scale in Fig. (5a) (black and white symbols). The latter are compared to the dilute expansions provided by Rubinstein and Torquato (1989) and Torquato (2002):

$$
\kappa^{A} \approx \kappa_{A, d i l}=\frac{2 R^{2}}{9(1-f)}, \quad \kappa^{B} \approx \kappa_{B, \text { dil }}=\frac{4 R^{2}}{9(1-f) \log ^{2}(1-f)}, \quad f \rightarrow 1
$$

which are valid for spherical obstacles and voids, respectively. The first-order corrections above are plotted in Fig. (5a) in blue (model A) and red (model B). The solid line in black represents the upper bound from Doi (1976) computed 


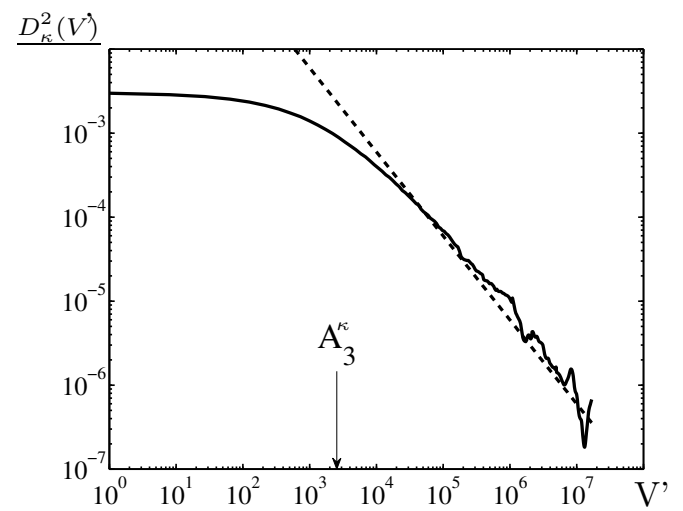

Fig. 3 Variance $D_{\kappa}^{2}\left(V^{\prime}\right)$ of the apparent permeability computed on non-overlapping subdomains of volume $V^{\prime}$, with respect to $V^{\prime}$, in number of voxels, in log-log scale. Dashed line: fit $\sim D_{\kappa}^{2} A_{3}^{\kappa} / V^{\prime}$ resulting from the scaling law (25).

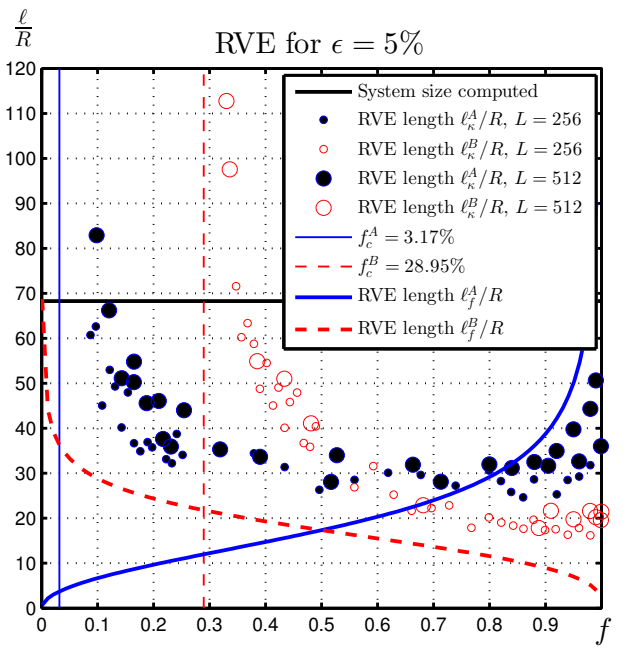

Fig. 4 Symbols: Normalized RVE lengths $\ell_{\kappa}^{A, B}$ for the permeability as a function of the pore volume fraction $f$, for models (A) and (B). The relative precision is fixed to $\epsilon_{\mathrm{rel}}=5 \%$. Thick lines: RVE lengths $\ell_{f}^{A, B}$ for the microstructure at the same precision. Horizontal black line: length $\ell$ of the most representative employed microstructure with $L^{3}=512^{3}$ voxels. Vertical lines: percolation thresholds $f_{c}^{A}$ and $f_{c}^{B}$. All lengths are given in units of spheres radius.

using the results of Rubinstein and Torquato (1989). The analytical expansion (28) is in good agreement with FFT data in the region $1-f<10^{-2}$. For $1-f>10^{-2}$, the effective permeability in model (A) is significantly lower than the dilute expansion and upper-bound. Similarly, the permeability of model (B) predicted by FFT computations is smaller than expansion (28) for $1-f>10^{-2}$. In the highly-dilute regime $1-f<10^{-2}$, the agreement between expansion (28) and FFT data for model (B) is not as good as for 


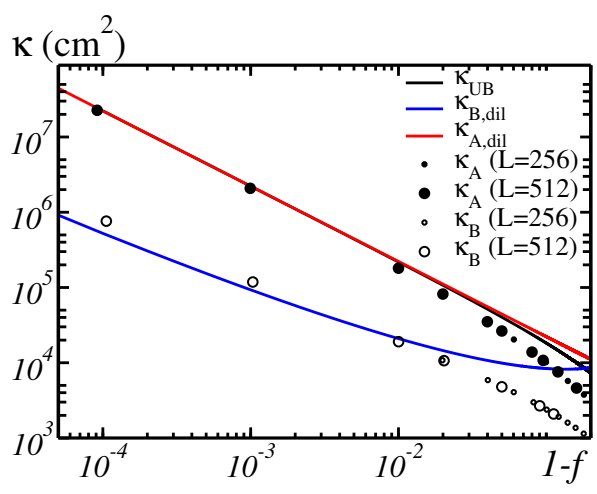

(a)

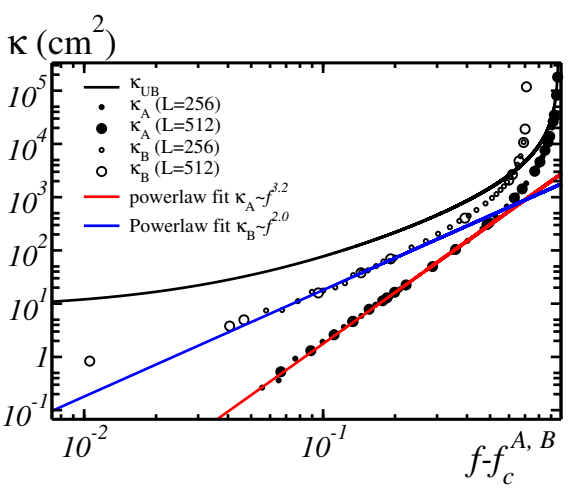

(b)

Fig. 5 Effective permeability $\kappa^{A, B}$ of models A (black disks) and B (white disks) vs. $1-f$, when $f \rightarrow 1$ (a) and $f-f_{c}^{A, B}$ when $f \gtrsim f_{c}^{A, B}$ is close to the percolation threshold (b). The FFT results were computed on grids of $256^{3}$ (small disks) and $512^{3}$ voxels (large disks). Solid lines: upper bound $\kappa^{U B}$ (black). (a): analytical results (28) for models A (red) and B (blue) and (b): powerlaw fits (29) of the FFT data for models A (blue) and B (red).

model (A). Remark however that particles in model (B) have highly elongated shapes so discretization effects should be important in this case. The obstacles surface/volume ratio is indeed, on average, $|\partial \gamma / \partial q| \rightarrow \infty$ when $q=1-f \rightarrow 0$ (Eq. 19).

The behavior of the permeability close to the percolation thresholds $f \rightarrow$ $f_{c}^{A}$ for model (A) and $f \rightarrow f_{c}^{B}$ for model (B) is represented in Fig. (5b). In the latter, the percolation thresholds are fixed to $f \rightarrow f_{c}^{A}=3.17 \%, f_{c}^{B}=28.95 \%$ and the permeability is plotted as a function of $f-f_{c}^{A, B}$ in $\log$-log scale. We consider volumes discretized on grids of $256^{3}$ and $512^{3}$ voxels and fit the data with power laws in a region of interest $\left|f-f_{c}^{A, B}\right|<0.2$ close to the percolation threshold. The latter are shown in blue $\left(256^{3}\right.$ voxels $)$ and red ( $512^{3}$ voxels $)$.

Numerical fits indicate:

$$
\kappa^{A} \sim 0.28 R^{2}\left(f-f_{c}^{A}\right)^{3.2}, \quad \kappa^{B} \sim 0.18 R^{2}\left(f-f_{c}^{A}\right)^{2.0}
$$

Predictions from the literature give $\kappa^{A} \sim\left(f-f_{c}^{A}\right)^{4}$ (Feng et al, 1987) for model (A). Likewise, an exponent equal to 4 was numerically determined by Martys et al (1994) for model (A), using spheres of diameter 15 voxels in a grid of $100^{3}$ voxels. In the same condition, with a larger grid we recovered an exponent of about 3.5. In the present work, the range of validity of the fits (29) is limited to at most one decade. Computations on larger systems are required to confirm the above results.

Numerical values for the exponents near the percolation threshold are rough estimates, due to the challenging numerical computations but indicate that the exponent in model (B) is significantly lower than in model (A), i.e. close to the percolation threshold, the permeability in model $\mathrm{B}$ increases at a faster rate when $f$ increases. 


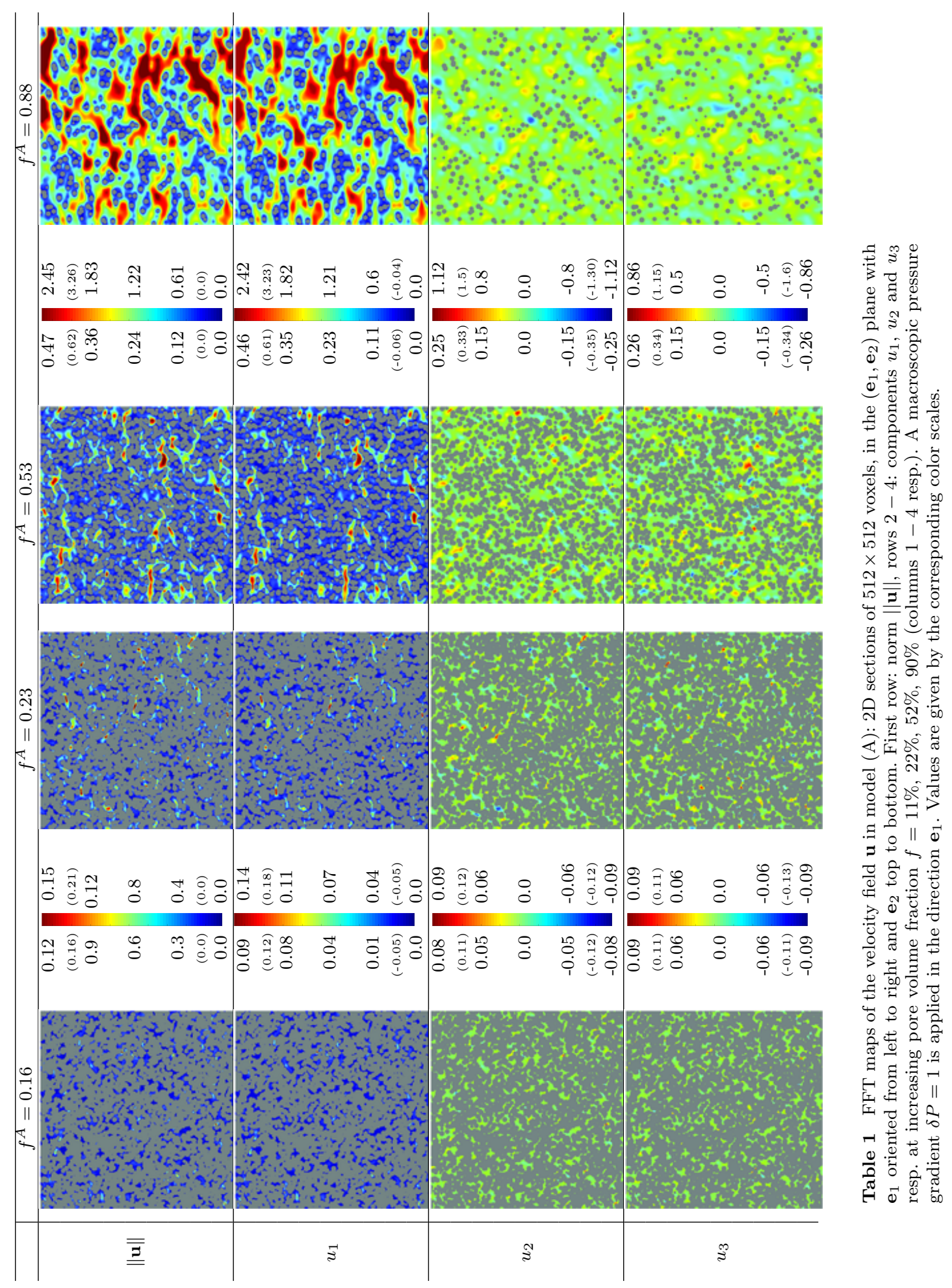




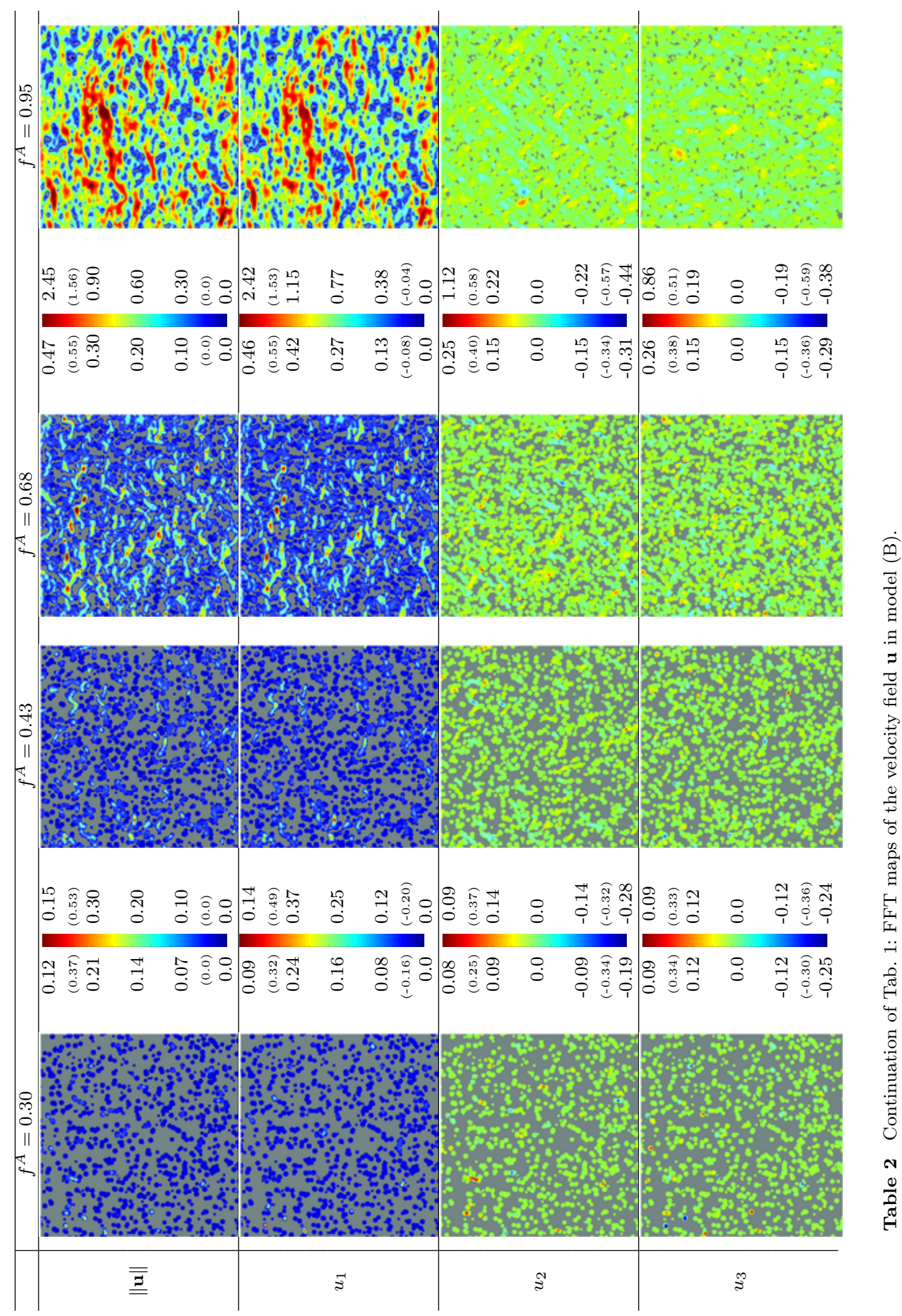




\section{Local fields}

In this section, are given and discussed maps of the velocity and pressure fields. The fields histogram and streamlines tortuosity are also studied.

\subsection{Maps and histograms of the velocity field}

FFT field maps are represented in Tabs. (1) and (2) for models (A) and (B) respectively. The maps are $512 \times 512$ voxels, 2 D sections along the plane $\left(\mathbf{e}_{1}, \mathbf{e}_{2}\right)$ where $\mathbf{e}_{1}$ is oriented right to left and $\mathbf{e}_{2}$ top to bottom. The norm $\|\mathbf{u}\|$ of the velocity vector as well as its components $u_{i}$ are shown at 4 fixed values of the pore volume fraction. The component $u_{1}$ is parallel to the applied pressure field whereas $u_{2}$ and $u_{3}$ are transverse components. To compare the field maps for different values of the pore volume fraction, the colour scale is given and the values are indicated on the corresponding side of the field. Blue and red correspond to the lowest and highest values respectively, with green and yellow in-between, whereas the solid phase is coloured in grey. To highlight the fields patterns, each map has been thresholded at $75 \%$ of its maximum values. The maximum and minimum values of the velocity field, prior to the thresholding, is given in small font along the colour scale.

At lowest pore volume fraction $f^{A}=11 \%$ in model (A), the velocity field is localized at a few bright spots, which appears in yellow in the $2 \mathrm{D}$ section in Tab. 1, column 1. As expected, the velocity field is small even in regions where the largest pores appear. These zones are, presumably, either disconnected from the percolating paths, or not well connected to them. At higher pore volume fraction $f^{A}=0.22 \%$, the density of hot spots increases (parallel component $u_{1}$, column 2). Furthermore, the velocity field in these spots is higher. In general, the maximum value of the parallel component $u_{1}$ increases with the pore volume fraction from $0.06\left(f^{A}=0.22 \%\right)$ to $3.08\left(f^{A}=11 \%\right)$. The minimal value, which is always negative, also increases with the pore volume fraction, i.e. streamlines going backward are increasingly rare events. At the highest pore volume fraction $f^{A}=0.90$, the velocity component $u_{1}$ is very low in regions comprising 2 or 3 obstacles close to one another (shown in dark blue). It is also significantly higher in a few large zones of the pores (shown in red). This suggests that, even at high pore volume fraction, only a fraction of the pores participates to the overall permeability. At the pore volume fraction $f^{A}=0.90$, the transverse components $u_{2}$ and $u_{3}$ are much more homogeneous than $u_{1}$.

The field patterns for the parallel component $u_{1}$ observed on the FFT maps is confirmed by the field histogram Fig. 6 (a). When $f=90 \%$, the field histogram $P_{u_{1}}$ is strongly non-symmetric. A local maximum field histogram is observed at $u_{1} \equiv 0$, corresponding to regions located around clusters of obstacles. A second local maximum is observed at higher values of the velocity field, $u_{1} \approx 0.6$. At lower pore volume fraction, the field histogram is non-symmetric. However, they contain one local maximum only at $u_{1}=0$. The histogram 


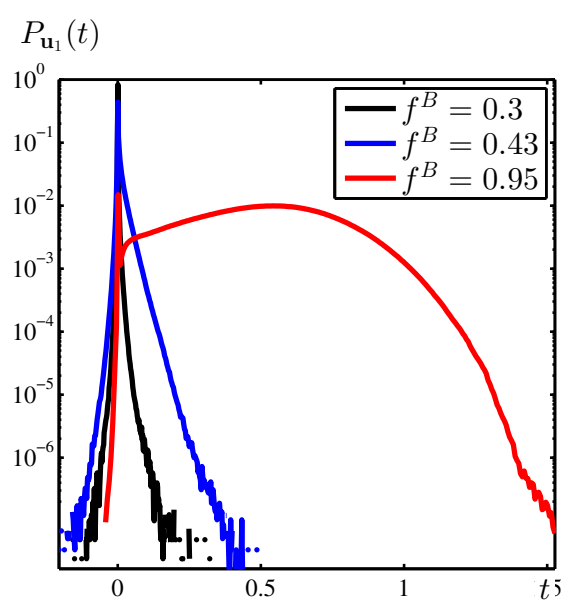

(a)

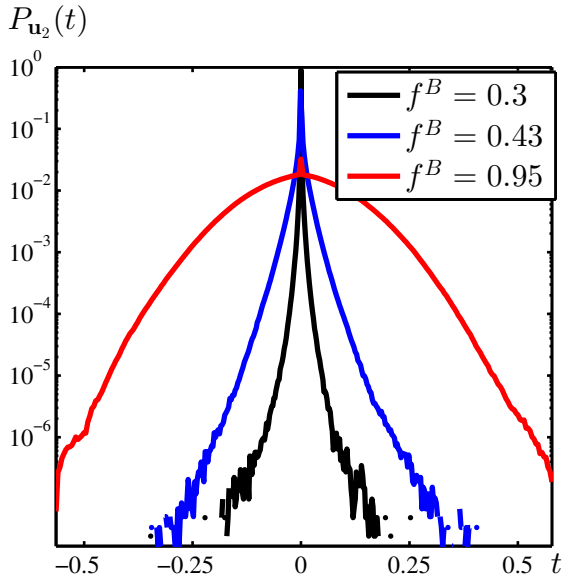

(b)

Fig. 6 (a) Histogram of the parallel component of the velocity field $u_{1}$ in model B, for three pore volume fraction, in lin-log plot. (b) Histogram of the transverse component of the velocity vector $u_{2}$ in model B for various pore volume fraction, in lin-log plot.

for the transverse component $u_{2}$, or equivalently, that of $u_{3}$ is represented in Fig. 6 (b). As for the parallel component, we observe a sharp peak around $u_{2}=0$ for the density of probability $P_{u_{2}}$. Except for the highest pore volume fraction $(f=0.95)$ on Fig. $6(\mathrm{~b})$, the transverse component shows a strongly non-Gaussian distribution of the velocity.

\subsection{Streamlines and orientation of the velocity field}

We now extract streamlines from the 3D velocity fields, defined as paths parallel to the velocity vector at each point. They span the microstructure domain from side (of equation $x_{1}=-L / 2$ ) to the opposite one (of equation $x_{1}=L / 2$ ). The streamlines's tortuosity $\tau$ is represented in Fig. (7a) for models (A) and (B) at increasing pore volume fraction and compared to the geometrical tortuosity $\tau_{\text {Geo }}^{\mathrm{A}, \mathrm{B}}$. Each point corresponds to one random configuration and different symbols are used for different system sizes. Dotted straight lines indicate the percolation threshold where $\tau=\infty$. For model (A), in the region close to the percolation threshold, around $f^{A}<30 \%$, the system sizes $L=256$ and $L=512$ used in this work are too small and the numerical results show strong variations. In any case, we expect the tortuosity to be a decreasing function of $f$. As shown in Fig. (7a) the streamlines's tortuosity for model (A) is only slightly lower than that of model (B), except when the pore volume fraction approaches the percolation threshold. As expected, lower tortuosity implies higher permeability. Indeed, the permeability of model (A) is higher than that of model (B), at given pore volume fraction $f$ (Fig. 2). Nevertheless the permeability in model (A) is often 2 or 3 times higher than that of (B), whereas 


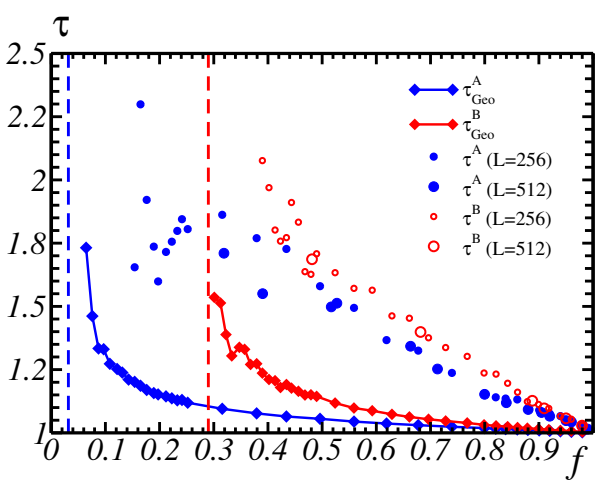

(a)

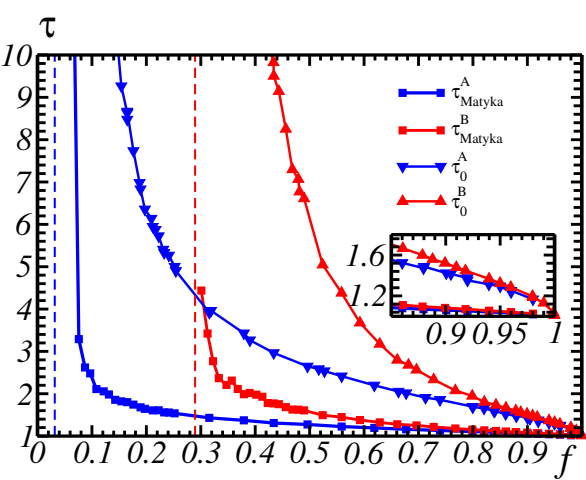

(b)

Fig. 7 (a) Tortuosity $\tau^{\mathrm{A}, \mathrm{B}}$ of the fluid streamlines computed using FFT data for models A (filled disks) and B (circles). Diamonds: geometrical tortuosity $\tau_{\mathrm{Geo}}^{\mathrm{A}, \mathrm{B}}$. (b) Viscuous tortuosity $\tau_{0}^{\mathrm{A}, \mathrm{B}}$ vs. porosity $f$ (triangles) for models A (blue) and B (red). Squares: tortuosity $\tau_{\mathrm{M}, \mathrm{B}}^{\mathrm{A}}$ defined by Eq. (17). Inner graph: close-up in the region $f \lesssim 1$.

the difference in terms of tortuosity is much smaller. For a given pore volume fraction, the morphological tortuosity, deduced from geodesic paths is much smaller than the tortuosity deduced from fluid flow characteristics. As a consequence the overall permeability $\kappa$ cannot be predicted directly from the geometrical tortuosity $\tau_{G e o}$.

The static viscuous tortuosity $\tau_{0}^{\mathrm{A}, \mathrm{B}}$ and that defined in Eq. (17) $\tau_{\text {Matyka }}^{\mathrm{A}, \mathrm{B}}$ are represented in Fig. (7b). They are computed as a function of the porosity $f$ for both models $\mathrm{A}$ and $\mathrm{B}$. The tortuosity $\tau_{0}^{\mathrm{A}, \mathrm{B}}$ is much higher than the streamlines tortuosity $\tau^{\mathrm{A}, \mathrm{B}}$, especially in the dilute regime $f \approx 1$.

The tortuous behavior of the velocity field at low pore volume fraction is confirmed by the covariance of orientation $C_{\alpha}(h)$ plotted in Fig. 8 for model (A). The latter decreases with $h$ at low pore volume fraction, and stays nearly constant for $h \geq h_{c}$, where $h_{c}$ depends on $f$. The length $h_{c}$ represents the typical size of regions where the velocity field is locally correlated due to viscosity. Beyond $h_{c}$, the finite covariance $C_{\alpha}(h)$ is a result of macroscopic coupling, i.e. the overall direction of the fluid follows that of the applied pressure gradient. As expected, the limiting value of $C_{\alpha}(h)$ increases with $f$, because of the tortuous path followed by the fluid near the percolation threshold, and $h_{c}$ increases with $f$, because the size of the channels followed by the fluid also increases with $f$. In the limit $f=1$, we expect $C_{\alpha}(h) \equiv 1$, a result consistent with Poiseuille flow.

\section{Conclusion}

In this numerical work, we used FFT-based full-fields computations for the Stokes flow to estimate the effective permeability of two simple 3D Boolean random models, where the fluid flows inside spheres or its complementary set. 


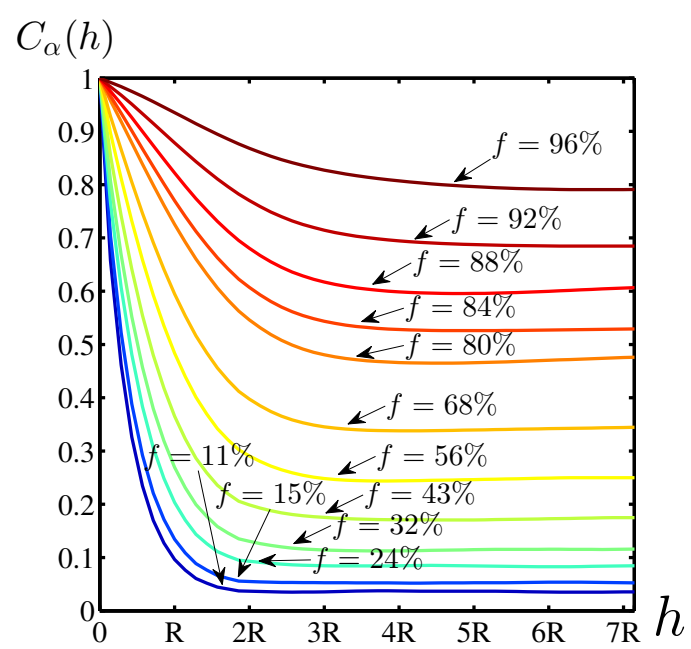

Fig. 8 Covariance of the orientation of the velocity field $C_{\alpha}(h)$ for model (A), for various increasing pore volume fraction $f$.

Very large system sizes were required to carry out computations, especially near the percolation points. For volume fractions $5 \%$ higher than the percolation threshold and a relative precision of $5 \%$ on the permeability, the size of the RVE becomes larger than 80 times the spheres's radius. No analytical formula was found to provide meaningful estimates of the effective permeability, for any considered model. Similar tortuosity ratios were observed for the fluid's streamlines, for both models, except in the vicinity of the percolation threshold. This is contrast with the geometrical tortuosity, which is significantly lower than that of the streamlines.

The effective permeability was found to be quite different for the two models, with the highest one recovered for spherical obstacles and corresponding to the lowest percolation threshold. Furthermore, as highlighted by the field maps and histograms, at moderate pore volume fraction, the velocity field is heterogeneous, and very small in regions located around or in-between clusters of obstacles. The velocity histograms were found to be non-Gaussian in general. These results confirm that the permeability, a highly structure-dependent property, is not only affected by the tortuosity but also by the distribution of the channels's size and surface areas.

Acknowledgements The research leading to the results presented has received funding from the European Union's Seventh Framework Programme (FP7 / 2007-2013) for the Fuel Cells and Hydrogen Joint Technology Initiative under grant agreement 303429. The authors thank an anonymous referee for useful remarks. 


\section{References}

Altendorf, H., Jeulin, D.: Stochastic modeling of a glass fiber reinforced polymer. In: Mathematical Morphology and Its Applications to Image and Signal Processing, Kluwer Academic, New-York, vol. 6671, pp. 439-450 (2011)

Azzimonti, D.F., Willot, F., Jeulin, D.: Optical properties of deposit models for paints: full-fields FFT computations and representative volume element. J. Mod. Optics 60(7), 519-528 (2013)

Belov, E.B., Lomov, S.V., Verpoest, I., Peters, T., Roose, D., Parnas, R.S., Hoes, K., Sol, H.: Modelling of permeability of textile reinforcements: lattice Boltzmann method. Composites Sc. Tech. 64(7-8), 1069-1080 (2004)

Bignonnet, F., Dormieux, L.: FFTbased bounds on the permeability of complex microstructures. Int. J. Num. An. Meth. Geomechanics 38(16), $1707-$ 1723 (2014)

Boutin, C.: Study of permeability by periodic and self-consistent homogenisation. Eur. J. Mech. A/Solids 19(4), 603-632 (2000)

Carman, P.C.: Fluid flow through granular beds. Trans.-Inst. Chem. Eng. 15, 150-166 (1937)

Cheng, H., Papanicolaou, G.: Flow past periodic arrays of spheres at low Reynolds number. J. Fluid Mech. 335, 189-212 (1997)

Childress, S.: Viscous flow past a random array of spheres. J. Chem. Phys. 56(6), 2527-2539 (1972)

Doi, M.: A new variational approach to the diffusion and the flow problem in porous media. J. Phys. Soc. Japan 40(2), 567-572 (1976)

Dormieux, L., Kondo, D., Ulm, F.J., Microporomechanics, John Wiley \& Sons, Chichester (2006)

$\mathrm{Du}, \mathrm{X}$., Ostoja-Starzewski, M.: On the size of representative volume element for Darcy law in random media. Proc. R. Soc. A 462(2074), 2949-2963 (2006)

Ene, I.H., Sanchez-Palencia, É.: Équations et phénomènes de surface pour lécoulement dans un modele de milieu poreux. Journal de mécanique 14(1), 73-108 (1975)

Happel, J.: Viscous flow in multiparticle systems: slow motion of fluids relative to beds of spherical particles. AIChE 4(2), 197-201 (1958)

Hinch, E.J.: An averaged-equation approach to particle intercations in a fluid suspension. J. Fluid Mech. 83(4), 695-720 (1977)

Howells, I.: Drag due to the motion of a Newtonian fluid through a sparse random array of small fixed rigid objects. J. Fluid Mech. 64(3), 449-476 (1974)

Jeulin, D., Moreaud, M.: Percolation dagrégats multi-échelles de sphères et de fibres - Application aux nanocomposites. Proc. Matériaux 2006, Dijon, 341348, www.archives-ouvertes.fr/hal-01163539, accessed June 12, 2015

Johnson, L.D., Plona, J.T., Scala, C., Pasierb, F., Kojima, H.: Tortuosity and acoustic slow waves. Phys. Rev. Let. 49(25), 1840-1844 (1982)

Johnson, D.L., Koplik, J.,Dashen, R.: Theory of dynamic permeability and tortuosity in fluid-saturated porous media. J. Fluid Mech. 176, 379-402 
(1987)

Kanit, T., Forest, S., Galliet, I., Mounoury, V., Jeulin, D. Determination of the size of the representative volume element for random composites: statistical and numerical approach. Int. J. Sol. Struct. 40(13-14), 3647-3679 (2003)

Karim, M.R., Krabbenhoft, K., Lyamin, A.V.: Permeability determination of porous media using large-scale finite elements and iterative solver. Int. J. Num. An. Meth. Geomechanics 38(10), 991-1012 (2014)

Koo, S., Sangani, A.S.: Effective-medium theories for predicting hydrodynamic transport properties of bidisperse suspensions. Physics of Fluids 14(10), 3522-3533 (2002)

Kozeny, J.: Ueber kapillare leitung des wassers im boden. Sitzungsber Akad Wiss Wien 136, 271-306 (1927)

Kuwabara, S.: The forces experienced by randomly distributed parallel circular cylinders or spheres in a viscous flow at small reynolds numbers. J. Phys. Soc. Japan 14(4), 527-532 (1959)

Lee, B.H., Lee S.K.: Effects of specific surface area and porosity on cube counting fractal dimension, lacunarity, configurational entropy, and permeability of model porous networks: Random packing simulations and NMR microimaging study. J. Hydrology 496, 122-141 (2013)

van der Marck, S.C.: Network approach to void percolation in a pack of unequal spheres. Phys. Rev. Lett. 77(9), 1785-1788 (1996).

Martin, J.J., McCabe, L.W., Monrad C.C.: Pressure drop through stacked spheres. Effect of orientation. Chem. Eng. Progress 47, 91-94 (1951).

Martys, N., Torquato, S., Bentz, D.: Universal scaling of fluid permeability for sphere packings. Phys. Rev. E 50(1), 403-408 (1994)

Matheron, G.: Remarques sur la loi de Darcy. École Nationale Supérieure des Mines, Note Géostatistique No. 59, www.cg.ensmp.fr/bibliotheque/ public/MATHERON_Rapport_00085.pdf (1965), accessed June 12, 2015

Matheron, G.: Genèse et signification énergétique de la loi de Darcy. Revue de l'institut français du pétrole et annales des combustibles liquides $\mathbf{2 1}(11)$, 1697-1706 (1966)

Matheron, G., Élements pour une theorie des milieux poreux, Paris, Masson (1967)

Matheron, G., The theory of regionalized variables and its applications. École Nationale Suprieure des Mines, Paris (1971)

Matyka M., Koza, Z.: How to Calculate Tortuosity Easily? In: AIP Conf. Proc. 1453, 17-22 (2012)

Monchiet, V., Bonnet, G., Lauriat, G.: A FFT-based method to compute the permeability induced by a Stokes slip flow through a porous medium. C. R. Mécanique 337(4), 192-197 (2009)

Nguyen, T.-K., Monchiet, V., Bonnet, G.: A Fourier based numerical method for computing the dynamic permeability of periodic porous media. Eur. J. Mech. B/Fluids 37, 90-98 (2013)

Perrot, C., Chevillotte, F., Panneton, R.: Dynamic viscous permeability of an open-cell aluminum foam: Computations versus experiments. J. Appl. Phys. 103(2), 024909 (2008) 
Peyrega, C., Jeulin, D.: Estimation of acoustic properties and of the representative volume element of random fibrous media. J. Appl. Phys. 113(10), 104901 (2013)

Prager, S.: Viscous flow through porous media. Phys. Fluids 4(12), 1477-1482 (1961)

Priour Jr., D.J.: Percolation through voids around overlapping spheres: a dynamically based finite-size scaling analysis. Phys. Rev. E 89(1), 012148 (2014)

Redenbach, C., Wirjadi, O., Rief, S., Wiegmann, A.: Modelling a ceramic foam for filtration simulation. Adv. Eng. Mat. 13(3), 171-177 (2011)

Richardson F.J., Zaki N.W.: The sedimentation of a suspension of uniform spheres under conditions of viscous flow. Chem. Eng. Sc. 3(2), 65-73 (1954)

Rintoul M.D., Torquato S.: Precise determination of the critical threshold and exponents in a three-dimensional continuum percolation model. J. Phys. A: Mathematical and General 30(16), 585-592 (1997)

Rubinstein J., Torquato S.: Flow in random porous media: mathematical formulation, variational principles, and rigorous bounds. J. Fluid Mech. 206, 25-46 (1989)

Saad, Y., Schultz, M.: GMRES: a generalized minimal residual algorithm for solving nonsymmetric linear systems. SIAM J. Sc. Stat. Comput. 7, 856-869 (1986)

Sangani A.S., Acrivos A.: Slow flow through a periodic array of spheres. Int. J. of Multiphase Flow 8(4), 343-360 (1982)

Feng, S., Halperin, B.I., Sen, P.N.: Transp. properties of continuum systems near the percolation threshold. Physical review B 35(1), 197-214 (1987)

Torquato, S.: Random heterogeneous materials: microstructure and macroscopic properties, Springer, New-York (2002)

Torquato S., Lu, B.: Rigorous bounds on the fluid permeability: Effect of polydispersivity in grain size. Physics of Fluids A: Fluid Dynamics 2(4), 487-490 (1990)

Weissberg, L.H., Prager, S.: Viscous flow through porous media. III. Upper bounds on the permeability for a simple random geometry. Phys. Fluids 13(12), 2958-2965 (1970)

Willot, F., Jeulin, D.: Elastic behavior of composites containing boolean random sets of inhomogeneities. Int. J. Eng. Sc. 47(2), 313-324 (2009)

Willot, F., Jeulin, D.: Elastic and electrical behavior of some random multiscale highly-contrasted composites. Int. J. Multiscale Comput. Eng. 9(3), 308-326 (2010)

Wiegmann, A.: Computation of the permeability of porous materials from their microstructure by FFF-Stokes. Berichte Fraunhofer ITWM 129, http:// kluedo.ub.uni-kl.de/files/1984/bericht129.pdf (2007), accessed 22 July 2015

Zick A.A., Homsy G.M.: Stokes flow through periodic arrays of spheres. J. Fluid Mech. 115, 13-26 (2006) 